\title{
Artificial Intelligence and Pet Imaging, Part I
}

Editors

BABAK SABOURY

ARMAN RAHMIM

ELIOT SIEGEL

\section{PET CLINICS}

www.pet.theclinics.com

Consulting Editor

ABASS ALAVI

October 2021 • Volume 16 - Number 4 